\title{
CORRELAÇÃO ENTRE PARÂMETROS DA CINÉTICA DE INTUMESCIMENTO COM CARACTERÍSTICAS ESTRUTURAIS E HIDROFÍLICAS DE HIDROGÉIS DE POLIACRILAMIDA E METILCELULOSE
}

\author{
Fauze A. Aouada \\ Departamento de Química, Universidade Federal de São Carlos, 13560-905 São Carlos - SP, Brasil \\ Edvani C. Muniz \\ Departamento de Química, Universidade Estadual de Maringá, 87020-900 Maringá - PR, Brasil \\ Carlos M. P. Vaz e Luiz H. C. Mattoso* \\ Laboratório Nacional de Nanotecnologia para o Agronegócio, Embrapa Instrumentação Agropecuária, \\ 13560-970 São Carlos - SP, Brasil
}

Recebido em 16/7/08; aceito em 16/1/09; publicado na web em 4/8/09

\begin{abstract}
CORRELATION BETWEEN PARAMETERS OF SWELLING KINETIC WITH NETWORK AND HYDROPHILIC CHARACTERISTICS OF POLYACRYLAMIDE AND METHYLCELLULOSE HYDROGELS. In this paper, the effects of acrylamide (AAm), methylcellulose (MC) contents, $\mathrm{pH}$ and ionic strength on kinetic, network and hydrophilic properties of polyacrylamide and methylcellulose hydrogels were investigated. The hydrogels were characterized by evaluating of network [average molecular weight between crosslinks $\left(\mathrm{M}_{\mathrm{C}}\right)$, crosslink density $(\mathrm{q})$ and the number of elastically effective chains $\left(\mathrm{V}_{\mathrm{e}}\right)$ ], and kinetic parameters [diffusional exponent $(n)$, diffusion constant $(k)$ and diffusion coefficient (D)]. Such properties were controlled by adjusting of the AAm, MC contents, $\mathrm{pH}$ and ionic strength factors. Due to high hydrophilicity and fast water-uptake, the PAAm-MC hydrogels can be considered as materials for potential applications in agricultural fields, mainly in controlled release of water or pesticides.
\end{abstract}

Keywords: hydrogels, kinetic properties, structural properties.

\section{INTRODUÇÃO}

Celulose é o mais abundante, renovável e biodegradável polissacarídeo encontrado na natureza. Mesmo tratando-se de um polímero hidrofílico, a celulose é insolúvel em água devido ao denso arranjo de suas cadeias. Tal empacotamento se deve às fortes interações intramoleculares que ocorrem por meio de ligações de hidrogênio. ${ }^{1} \mathrm{~A}$ partir de um simples tratamento alcalino, com solução de hidróxido de sódio seguido de uma reação com cloreto de metila, ${ }^{2}$ pode-se substituir uma fração de grupamentos hidroxilas por grupamentos metilas. Essa substituição diminui as interações intramoleculares e o empacotamento das cadeias. O principal produto desse tratamento é a metilcelulose (MC). Os grupamentos metilas presentes na estrutura da MC dificultam um arranjo mais ordenado das cadeias, provocando mudanças significativas nas propriedades físico-químicas em relação à celulose. Por exemplo, a metilcelulose apresenta grande solubilidade em água, enquanto que a celulose é insolúvel em água devido à sua cristalinidade. $^{3}$

Hidrogéis podem ser definidos como sistemas hidrofílicos formados por dois ou mais componentes unidos por ligações covalentes e/ou eletrostáticas dispostos em uma ou mais redes tridimensionalmente estruturadas ${ }^{4,5}$ envoltas por moléculas de um determinado solvente, geralmente água. A quantidade de água adsorvida pelos hidrogéis geralmente está relacionada com a hidrofilicidade das cadeias e a densidade de agente de reticulação ${ }^{6,7}$ utilizada na síntese.

Dependendo da natureza (neutro ou iônico) dos componentes que originam os hidrogéis ou de substâncias incorporadas à matriz, $\mathrm{o}$ hidrogel pode responder diferentemente ao se alterar as características do meio externo, tais como $\mathrm{pH}^{8}{ }^{8}$ força iônica ${ }^{9}$ ou da quantidade de algum analito químico específico, por exemplo, corantes, ${ }^{10,11}$ pesticidas e ou nutrientes. ${ }^{12,13}$

\footnotetext{
*e-mail: mattoso@cnpdia.embrapa.br
}

O controle dos fatores tais como densidade de cadeias poliméricas, quantidade de agente de reticulação e balanço hidrofílicohidrofóbico possibilita a obtenção de matrizes com propriedades mecânicas ${ }^{14}$ e estruturais ${ }^{15}$ ajustadas para um grau de intumescimento adequado para uma determinada aplicação. ${ }^{16,17}$

A metilcelulose foi adicionada na solução de síntese juntamente com a acrilamida com o intuito de obter hidrogéis com maior hidrofilicidade, devido à presença de grupamentos hidroxilas presentes na metilcelulose. Além disso, a possibilidade de sintetizar hidrogéis com teores de MC proporciona a obtenção de hidrogéis com custos menores e mais biodegradáveis, visto que, além da metilcelulose ser um polissacarídeo biodegradável possui um custo inferior ao da acrilamida que se trata um produto sintético.

$\mathrm{O}$ entendimento do efeito de ambientes externos ( $\mathrm{pH}$ e força iônica, entre outros) na cinética de intumescimento e nas propriedades estruturais e hidrofílicas de hidrogéis e também a relação destes aspectos com as concentrações de acrilamida e de metilcelulose na solução formadora dos hidrogéis motivaram o desenvolvimento deste trabalho. Foram estudadas cinéticas de intumescimento de hidrogéis com enorme potencial na utilização como veículo carreador em sistemas de liberação controlada de água e de insumos agrícolas. Foram avaliados parâmetros da cinética de intumescimento [expoente difusional $(n)$, constante de difusão $(k)$ e coeficiente de difusão (D)] e parâmetros estruturais [massa molar média entre retículos $\left(\mathrm{M}_{\mathrm{C}}\right)$, densidade de reticulação (q) e número de cadeias efetivamente elásticas por unidade de volume $\left(\mathrm{V}_{\mathrm{e}}\right)$ ], que foram obtidos a partir de curvas de grau de intumescimento em função do tempo. Para isso, foram preparados hidrogéis com diferentes teores de acrilamida (AAm) e metilcelulose (MC), os quais foram investigados em diferentes meios de intumescimento ( $\mathrm{pH}$ e força iônica). Os resultados permitiram correlacionar a cinética de intumescimento com a estrutura e a hidrofilicidade dos hidrogéis. 


\section{PARTE EXPERIMENTAL}

\section{Síntese dos hidrogéis}

Os hidrogéis constituídos de poliacrilamida (PAAm) e do polissacarídeo biodegradável metilcelulose $\left(\mathrm{MC}, \mathrm{M}_{\mathrm{n}}=40.000 \mathrm{~g} \mathrm{~mol}^{-1}\right.$, viscosidade $400 \mathrm{cP}$, com 27,5-31,5\% de grupamentos metilas, 68,5$72,5 \%$ de grupamentos hidroxilas, dados fornecidos pelo fabricante Aldrich) foram obtidos por meio de polimerização química do monômero acrilamida (AAm, Fluka) com concentrações de 3,6; 7,2, 14,7 e $21,7 \% \mathrm{~m} / \mathrm{v}$ em solução aquosa contendo MC com concentrações de 0,5 e $1,0 \% \mathrm{~m} / \mathrm{v}$ e do agente de reticulação N'-N-metilenobisacrilamida (MBAAm, Aldrich) à $8,55 \mu \mathrm{mol} \mathrm{mL}^{-1}$. A concentração de N,N,N',N'- tetrametil-etilenodiamina (TEMED, Sigma), utilizado como catalisador, foi mantida constante em 3,21 $\mu \mathrm{mol} \mathrm{mL}^{-1}$. Depois de preparar a mistura, borbulhou-se $\mathrm{N}_{2}$ na solução durante 20 minutos para a eliminação de oxigênio. Persulfato de sódio $\left(\mathrm{Na}_{2} \mathrm{~S}_{2} \mathrm{O}_{8}\right.$, Sigma $)$ $\left(3,38 \mu \mathrm{mol} \mathrm{mL} \mathrm{mL}^{-1}\right)$ foi adicionado com intuito de iniciar a reação de polimerização que ocorre via radical livre. ${ }^{18}$

\section{Cinética de intumescimento}

As propriedades hidrofílicas dos hidrogéis foram estudadas por medidas de grau de intumescimento $(\mathrm{Q})$ em função do tempo em diferentes meios de intumescimento. Os estudos de intumescimento foram realizados em soluções com diferentes $\mathrm{pH}(4,0,7,0$ ou 10,0) ou em pH 7,0 mas com força iônica variável $(0,025 ; 0,10$ e 0,20 $\left.\mathrm{mol} \mathrm{L} \mathrm{L}^{-1}\right)$. As soluções foram preparadas de acordo com procedimento descrito por Morita e Assumpção. ${ }^{19}$ Para a solução com $\mathrm{pH}=4,0$ : $95,0 \mathrm{~mL}$ de solução glicocol $0,10 \mathrm{~mol} \mathrm{~L}^{-1}$ e 5,0 mL de $\mathrm{HCl} 1,0 \mathrm{~mol}$ $\mathrm{L}^{-1}$; para a solução com $\mathrm{pH}=10,0: 60,0 \mathrm{~mL}$ de solução glicocol $0,10 \mathrm{~mol} \mathrm{~L}^{-1}$ e $40,0 \mathrm{~mL}$ de $\mathrm{NaOH} 1,0 \mathrm{~mol} \mathrm{~L}^{-1} ; \mathrm{pH}=7,0$ foi utilizada água destilada. A influência da força iônica no intumescimento dos géis foi avaliada em $\mathrm{pH}$ 7,0. A força iônica no meio de intumescimento foi ajustada utilizando $\mathrm{NaCl}$ nas concentrações de 0,025 ; 0,10 e $0,20 \mathrm{~mol} \mathrm{~L}^{-1}$.

Os hidrogéis depois de intumescidos e em equilíbrio foram cortados em forma circular utilizando um molde (diâmetro de 2,6 $\mathrm{cm})$ construído em aço inox e secos em estufa com temperatura controlada de $25,0 \pm 0,1^{\circ} \mathrm{C}$. A massa média dos hidrogéis secos utilizados nesse estudo foi aproximadamente $150 \mathrm{mg}$. Para determinações de Q, primeiramente os hidrogéis secos foram pesados em uma balança analítica e posteriormente colocados para intumescer em $20 \mathrm{~mL}$ de meio. Para cada medida correspondente a um tempo " $\mathrm{t}$ ", os hidrogéis foram retirados do meio de intumescimento, sendo as superfícies secas para remover excesso de água e depois novamente pesados.

Os valores de $\mathrm{Q}$ dos diferentes hidrogéis foram calculados a partir da Equação 1:20,21

$$
Q \%=\frac{M_{t}-M_{0}}{M_{0}} x 100
$$

onde $\mathrm{M}_{\mathrm{t}}$ é a massa do hidrogel intumescido em um tempo t e $\mathrm{M}_{0}$ é a massa desse mesmo hidrogel, após secagem.

\section{Parâmetros da cinética de intumescimento}

Alguns parâmetros da cinética de intumescimento foram obtidos por meio de medidas de grau de intumescimento em função do tempo (F vs t) em soluções de diferentes valores de $\mathrm{pH}: 4,0 ;$ 7,0 e 10,0. Para cada curva de $\mathrm{F} v s \mathrm{t}$, o expoente difusional $(n)$ e constante de difusão $(k)$ foram calculados utilizando a Equação 2:22,23

$$
F=\frac{M_{t}}{M_{e q}}=k t^{n}
$$

onde $\mathrm{M}_{\mathrm{eq}}$ é a massa do hidrogel no equilíbrio de intumescimento, t é o tempo. $k$ é conhecida como constante de difusão e depende do tipo do hidrogel e do meio de intumescimento.

A Equação (2) pode ser aplicada desde os estágios iniciais até 60 $\%$ da curva de $\mathrm{F} v s \mathrm{t} .{ }^{24} \mathrm{O}$ aumento do grau de intumescimento com o tempo é praticamente linear, e após o estágio de $60 \%$, o intumescimento não segue mais essa tendência, ou seja, praticamente o grau de intumescimento não sofre mais variação com o tempo (atingindo o estado de equilíbrio). É importante ressaltar, que em curvas com coeficiente de regressão linear $\left(\mathrm{R}^{2}\right)$ inferiores ao apresentado na Figura $2\left(R^{2}=0.9994\right)$, a diferença entre os patamares (lineares e não lineares) são mais acentuados. O que mostra que realmente a Equação 2 é somente válida para estágios $\approx 60 \%$.

Para o cálculo de $n$ e de $k$ faz-se um gráfico de $\ln \mathrm{F} v s \ln \mathrm{t}$. Sendo que o valor do expoente difusional $n$ é o coeficiente angular da curva e o coeficiente linear é $\ln k$.

O estudo de difusão do solvente para o interior dos hidrogéis foi realizado através das análises dos valores do coeficiente de difusão $D$. Os valores de $D$ foram obtidos através da Equação 3:25,26

$D=\pi r^{2}\left(\frac{k}{4}\right)^{1 / n}$

onde $D$ é dado em $\mathrm{cm}^{2} \mathrm{~s}^{-1}, \mathrm{r}$ é o raio do hidrogel seco (dado em centímetros) em forma cilíndrica. Para cada ensaio, o valor de $\mathrm{r}$ foi medido antes de se iniciarem os experimentos. Os valores de $n$ e $k$ foram obtidos utilizando a Equação 2.

\section{Estudo dos parâmetros estruturais de redes dos hidrogéis}

Normalmente, os parâmetros grau de intumescimento (Q) e massa molar média entre retículos $\left(\mathrm{M}_{\mathrm{C}}\right)$ são os mais utilizados para caracterizar uma rede polimérica tridimensional. Outros parâmetros tais como densidade de reticulação (q), que é proporcional à fração molar de agente de reticulação utilizado, e número de cadeias efetivamente elásticas por unidade de volume, simbolizado por $\mathrm{V}_{\mathrm{e}},{ }^{27}$ também são importantes para a caracterização estrutural da rede tridimensional de um hidrogel.

Neste trabalho, os valores de $\mathrm{M}_{\mathrm{C}}$ foram obtidos utilizando-se a Equação $4:^{28}$

$M_{C}=-V_{l} d_{P} \frac{\left(V_{S}^{1 / 2}-V_{S / 2}\right)}{\ln (1)-V_{S}+V_{S}+x V_{S}^{2}}$

onde $\mathrm{V}_{1}$ é o volume molar do solvente (água) $\left(\mathrm{mL} \mathrm{mol}^{-1}\right)$, $\mathrm{d}_{\mathrm{p}}$ é a densidade do polímero (PAAm) $\left(\sim 1,10 \mathrm{~g} \mathrm{~mL}^{-1}\right), \mathrm{V}_{\mathrm{s}}$ é a fração de volume do gel intumescido (igual a $1 / F$ ) e $\chi$ é o parâmetro de interação de entre solvente e polímero $(\sim 0,466){ }^{29}$

Os valores de $\mathrm{q}$ e $\mathrm{V}_{\mathrm{e}}$ foram calculados pelas Equações 5 e 6 :

$q=\frac{M_{0}}{M_{C}}$

onde $\mathrm{M}_{\mathrm{o}}$ representa a massa molar da unidade repetitiva.

$V_{e}=\frac{d_{P} N_{A}}{M_{C}}$

onde $\mathrm{N}_{\mathrm{A}}$ é o número de Avogadro. 


\section{RESULTADOS E DISCUSSÃO}

\section{Cinética de intumescimento}

A teoria do intumescimento foi desenvolvida por Flory e Rehner em 1943, e consideraram que o fenômeno de intumescimento é controlado basicamente por três forças: i) a variação de entropia pela mistura solvente-polímero; ii) a variação de entropia conformacional causada pela redução no número de conformações das cadeias, em consequiência de seu estiramento e iii) a entalpia de mistura do solvente e polímero. ${ }^{30}$ Assim, o intumescimento de um dado polímero é dependente do grau de interação entre as moléculas de solvente e do polímero, que pode ser relacionado com o parâmetro de interação solvente/polímero, $\chi$.

Os valores de grau de intumescimento no estado de equilíbrio $\left(\mathrm{Q}_{\mathrm{eq}}\right)$ obtidos a partir de curvas de cinética de intumescimento $(\mathrm{Q} v s$ t) para hidrogéis com diferentes teores de AAm e MC estão listados na Tabela 1 onde também são apresentados os valores de $n, k$ e $D$.

Na Figura 1 é apresentada a dependência do grau de intumescimento com o tempo de imersão para três diferentes hidrogéis em $\mathrm{pH}$
7,0, a $25^{\circ} \mathrm{C}$. Para cada curva de $\mathrm{Q}$ vs t o valor de $\mathrm{Q}_{\mathrm{eq}}$ foi coletado no tempo de imersão a partir do qual não se observou variação da massa do hidrogel por um período mínimo de 24 horas. Pode-se observar que os hidrogéis atingem equilíbrio em aproximadamente 10 horas. As curvas de Q vs $\mathrm{t}$ apresentam dois comportamentos distintos característicos de hidrogéis: no início o processo de intumescimento ocorre rapidamente. Mas o intumescimento passa a ser mais lento em um tempo de imersão próximo de atingir o equilíbrio. $\mathrm{O}$ hidrogel constituído por 3,6 \% de AAm e 1,0\% de MC apresenta valor Q de $2000 \%$ em apenas 1 hora após ser imerso em solução de pH 7,0 e após 10 horas de imersão nesta mesma solução o valor de Q é $9200 \%$.

Os valores de $\mathrm{Q}_{\mathrm{eq}}$ dos hidrogéis diferem significativamente quando se varia o tipo de hidrogel. Foi verificado que os valores de $Q_{\text {eq }}$ são fortemente influenciados tanto pela concentração de AAm quanto pela concentração de MC da solução em que o hidrogel foi obtido. Ao comparar, por exemplo, hidrogéis sintetizados com solução de mesma concentração de AAm (3,6 \%), observa-se que o valor de $\mathrm{Q}_{\mathrm{eq}}$ aumenta de $6400 \%$ para $9200 \%$, quando a concentração de MC é variada de 0,5 para $1,0 \%$. Isto indica um aumento na hidrofilicidade

Tabela 1. Parâmetros obtidos de cinética de intumescimento dos diferentes hidrogéis em soluções com diferentes valores de pH: 4,0, 7,0 e 10,0 , a $25^{\circ} \mathrm{C}$

\begin{tabular}{|c|c|c|c|c|c|}
\hline \multicolumn{6}{|c|}{$\mathrm{pH}=4,0$} \\
\hline & $\mathrm{Q}_{\mathrm{eq}}(\%)$ & $n$ & $D\left(10^{-5} \mathrm{~cm}^{2} \mathrm{~s}^{-1}\right)$ & $k\left(\mathrm{~s}^{-1}\right)$ & $\mathrm{R}^{2}$ \\
\hline $3,6 \%$ AAm e $0,5 \%$ MC & 3900 & $0,44 *$ & $4,46 *$ & $0,315 *$ & $0,9839 *$ \\
\hline $7,2 \%$ AAm e $0,5 \%$ MC & 2000 & 0,42 & 1,73 & 0,169 & 0,9996 \\
\hline $14,7 \%$ AAm e $0,5 \% \mathrm{MC}$ & 1300 & 0,39 & 1,20 & 0,137 & 0,9969 \\
\hline $21,7 \%$ AAm e $0,5 \% \mathrm{MC}$ & 1200 & 0,38 & 1,92 & 0,215 & 0,9931 \\
\hline $3,6 \%$ AAm e $1,0 \%$ MC & 3200 & 0,52 & 2,78 & 0,092 & 0,9989 \\
\hline $7,2 \%$ AAm e $1,0 \% \mathrm{MC}$ & 2000 & 0,45 & 2,79 & 0,106 & 0,9984 \\
\hline $14,7 \%$ AAm e $1,0 \% \mathrm{MC}$ & 1400 & 0,40 & 2,60 & 0,159 & 0,9989 \\
\hline $21,7 \%$ AAm e $1,0 \% \mathrm{MC}$ & 1100 & 0,36 & 0,86 & 0,147 & 0,9971 \\
\hline \multicolumn{6}{|c|}{$\mathrm{pH}=7,0$} \\
\hline $3,6 \%$ AAm e $0,5 \%$ MC & 6400 & $0,60 *$ & $53,30 *$ & $0,616 *$ & $0,9681 *$ \\
\hline $7,2 \%$ AAm e $0,5 \% \mathrm{MC}$ & 2700 & 0,53 & 1,38 & 0,063 & 0,9995 \\
\hline $14,7 \%$ AAm e $0,5 \% \mathrm{MC}$ & 1700 & 0,45 & 2,32 & 0,104 & 0,9994 \\
\hline $21,7 \%$ AAm e $0,5 \% \mathrm{MC}$ & 1300 & 0,41 & 1,73 & 0,132 & 0,9994 \\
\hline $3,6 \%$ AAm e $1,0 \% \mathrm{MC}$ & 9200 & $0,78 *$ & $23,30 *$ & $0,030 *$ & $0,9775 *$ \\
\hline $7,2 \%$ AAm e $1,0 \% \mathrm{MC}$ & 3600 & 0,60 & 9,03 & 0,058 & 0,9988 \\
\hline $14,7 \%$ AAm e $1,0 \% \mathrm{MC}$ & 2000 & 0,49 & 2,31 & 0,078 & 0,9980 \\
\hline $21,7 \%$ AAm e $1,0 \% \mathrm{MC}$ & 1400 & 0,43 & 2,75 & 0,097 & 0,9994 \\
\hline \multicolumn{6}{|c|}{$\mathrm{pH}=10,0$} \\
\hline $3,6 \%$ AAm e $0,5 \% \mathrm{MC}$ & 5450 & $0,36 *$ & $12,60 *$ & $0,905 *$ & $0,9858 *$ \\
\hline $7,2 \%$ AAm e $0,5 \% \mathrm{MC}$ & 2530 & 0,44 & 2,91 & 0,156 & 0,9986 \\
\hline $14,7 \%$ AAm e $0,5 \% \mathrm{MC}$ & 1680 & 0,38 & 2,90 & 0,192 & 0,9981 \\
\hline $21,7 \%$ AAm e $0,5 \%$ MC & 1340 & 0,38 & 0,60 & 0,165 & 0,9981 \\
\hline $3,6 \%$ AAm e $1,0 \% \mathrm{MC}$ & 6110 & 0,55 & 0,77 & 0,063 & 0,9954 \\
\hline $7,2 \%$ AAm e $1,0 \% \mathrm{MC}$ & 2620 & 0,45 & 2,60 & 0,110 & 0,9971 \\
\hline $14,7 \%$ AAm e $1,0 \% \mathrm{MC}$ & 1550 & 0,39 & 2,53 & 0,135 & 0,9959 \\
\hline $21,7 \%$ AAm e $1,0 \% \mathrm{MC}$ & 1220 & 0,37 & 0,83 & 0,127 & 0,9963 \\
\hline
\end{tabular}

* os valores dos parâmetros podem não condizer com os valores reais, pois $\mathrm{R}^{2}<0.99$. 
com o teor de MC no hidrogel. Isto pode ser atribuído ao aumento da quantidade de grupamentos hidroxilas provenientes das cadeias de metilcelulose. Por outro lado, observa-se uma diminuição bastante significativa no valor de $\mathrm{Q}_{\mathrm{eq}}$ [de $3200 \%$ para $1200 \%(\mathrm{pH} 4,0)$; de $6400 \%$ para $1300 \%(\mathrm{pH} \mathrm{7,0)}$ e de $5450 \%$ para $1340 \%(\mathrm{pH} 10,0)]$ quando a concentração de AAm na solução formadora do hidrogel passa de 3,6 para 21,7 \%, mantendo a concentração de MC constante em $0,5 \%$. Isto indica que cadeias de MC são mais hidrofílicas que as cadeias de poliacrilamida. Como conseqüência, redes mais ricas em poliacrilamida, relativamente à quantidade de $\mathrm{MC}$, possuem menor capacidade de intumescimento, ${ }^{31} \mathrm{o}$ que contribui para baixos valores de $\mathrm{Q}_{\mathrm{eq}}$.

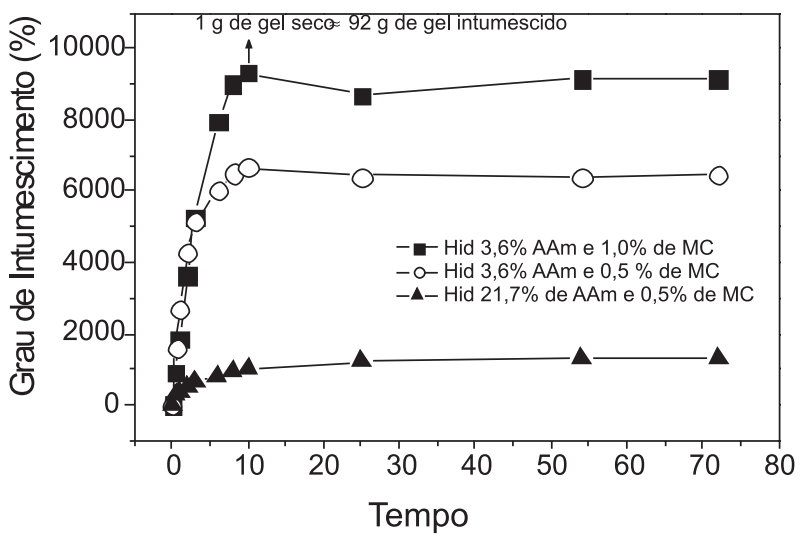

Figura 1. Curvas de $Q$ vs t para os hidrogéis sintetizados em solução com as concentrações indicadas de AAm e de MC. As medidas foram realizadas a $25^{\circ} \mathrm{C}, \mathrm{pH}=7,0$

\section{Parâmetros associados à cinética de intumescimento}

Os parâmetros associados à cinética de intumescimento são de extrema importância no entendimento mecanístico do processo de difusão da água para o interior dos hidrogéis. A partir das curvas de $\mathrm{F}$ vs $\mathrm{t}$ obtidas para hidrogéis com diferentes teores de AAm e MC e em diferentes meios de intumescimento (diferentes $\mathrm{pH}$ ) foi possível obter, para cada curva, os valores de $n$ e de $k$ (Equação 2) e do coeficiente de difusão $(D)$ (Equação 3). A determinação dos valores de $n$, de $k$ e de $D$ e a correlação destes com os respectivos valores de $\mathrm{Q}_{\text {eq }}$ são discutidas detalhadamente a seguir.

\section{Determinação do expoente difusional (n), constante de difusão} (k) e do coeficiente de difusão (D)

Para hidrogéis em formato cilíndrico, valores de $n$ entre 0,45 e 0,50 correspondem à difusão Fickiana. Valores de $n$ fora deste intervalo indicam que a difusão é do tipo não Fickiana. Um valor de $n \approx 1,0$ indica que a difusão do solvente para o interior do hidrogel ocorre por relaxamento das cadeias que compõem a rede. Em adição, para $0,5<n<1,0$ a difusão ocorre por transporte anômalo. No caso de transporte anômalo, o processo de difusão é governado, ao mesmo tempo, por difusão e pela relaxação das cadeias do hidrogel..$^{32,33}$

Na Figura 2 é mostrada a curva de $\ln \mathrm{F}$ vs $\ln \mathrm{t}$ em $\mathrm{pH}$ 7,0 utilizada para obtenções do expoente difusional $(n)$, constante de difusão $(k), \mathrm{e}$ coeficiente de difusão $(D)$ (utilizando a Equação 3), para o hidrogel preparado a partir de solução contendo $21,7 \%$ de AAm e $1,0 \%$ de MC. Curvas similares à apresentada na Figura 2 foram obtidas para intumescimento deste hidrogel em pH 4,0 e 10,0. Da mesma forma, curvas análogas foram obtidas para os demais hidrogéis nos três diferentes meios de intumescimento. Cabe ressaltar que os valores de $n$ e de $k$ foram obtidos, respectivamente, através dos coeficientes angulares e lineares de curvas similares à apresentada na Figura 2.

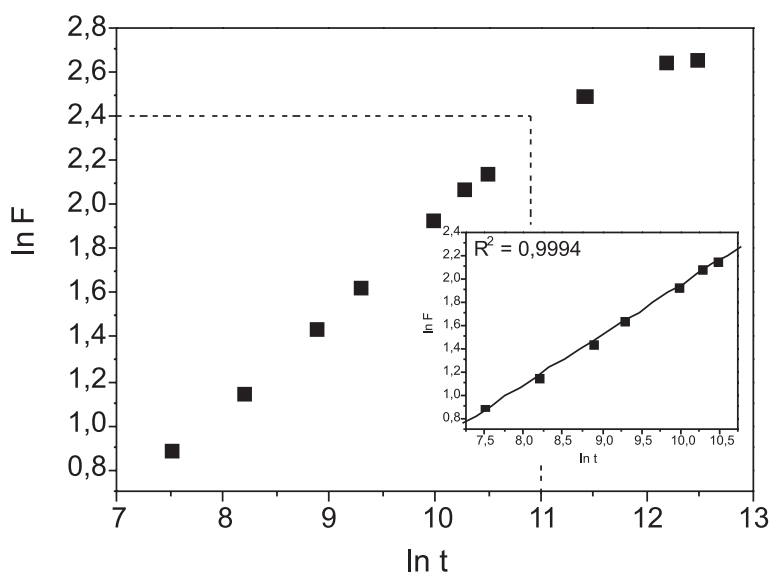

Figura 2. Curva de ln $F$ vs $\ln (t)$ a partir da qual foram obtidos os valores do expoente difusional ( $n$ ) e constante de difusão $(k)$ em $\mathrm{pH}$ 7,0 para o hidrogel sintetizado a partir de solução com 21,7\% de AAm e 1,0\% de MC

Na Tabela 1 são apresentados os valores de $n, k$ e $D$ para os diferentes hidrogéis ao serem intumescidos em soluções de $\mathrm{pH} 4,0$; 7,0 e 10,0. Pode-se perceber que para hidrogéis com um teor fixo de MC o aumento da concentração de AAm na solução formadora do hidrogel provoca diminuição significativa dos valores de $n$. Por outro lado, quando se fixa a concentração de AAm, os hidrogéis contendo maiores teores de MC apresentam maiores valores de $n$. Assim, pode-se inferir que o parâmetro $n$ é diretamente proporcional ao grau de intumescimento dos hidrogéis. Maiores valores de $n$ são observados para hidrogéis com os mais altos valores de $\mathrm{F}_{\mathrm{eq}}$, i.e., os hidrogéis sintetizados a partir de solução contendo 3,6 \% em AAm e 0,5 ou $1,0 \%$ em MC.

Os valores do coeficiente de difusão $D$ seguem a mesma tendência dos valores de $n$. Por exemplo, se compararmos os diferentes hidrogéis com $0,5 \%$ de $\mathrm{MC}$ intumescidos em $\mathrm{pH}=7,0$, observa-se que o valor de $D$ dos hidrogéis sintetizados com solução 3,6 \% em AAm $\left(D=53,30 \times 10^{-5} \mathrm{~cm}^{2} \mathrm{~s}^{-1}\right)$ é em torno de 31 vezes maior que para os hidrogéis sintetizados com 21,7\% em AAm $\left(D=1,73 \times 10^{-5} \mathrm{~cm}^{2} \mathrm{~s}^{-1}\right)$. Nos hidrogéis com $1,0 \%$ de MC os valores de $D$ variam 8 vezes [de $23,30 \times 10^{-5} \mathrm{~cm}^{2} \mathrm{~s}^{-1}\left(3,6 \%\right.$ em AAm) para $2,75 \times 10^{-5} \mathrm{~cm}^{2} \mathrm{~s}^{-1}(21,7 \%$ em AAm)]. De maneira geral, a difusão do solvente para o interior dos hidrogéis com 1,0\% de MC é mais rápida que nos hidrogéis com $0,5 \%$ de $\mathrm{MC}$ devido à maior quantidade de grupamentos hidroxilas existentes nas cadeias de MC. Alguns dos valores de $D$ obtidos para $\mathrm{pH}=7,0$ são menores que o valor de $D_{\mathrm{H} 20}$ a $25^{\circ} \mathrm{C}\left(=2,03 \times 10^{-5} \mathrm{~cm}^{2}\right.$ $\left.\mathrm{s}^{-1}\right)$. É importante ressaltar que ao passarem por sucessivos processos de intumescimento/secagem esses hidrogéis não perdem sua forma e nem sua estabilidade, apesar de o hidrogel intumescido ser constituído por aproximadamente 90-95\% de água. Assim, quando o hidrogel é colocado em contato com o meio de intumescimento, suas cadeias são rapidamente solvatadas formando uma primeira camada constituída por moléculas de água. Dessa forma, as moléculas de água no interior do hidrogel terão valores de energia e entropia diferenciados de acordo com a posição que se encontrar. Isto explica os baixos valores de $D$ observados para o intumescimento dos hidrogéis. No entanto, os hidrogéis sintetizados com $3,6 \%$ de AAm e $0,5 \%$ de MC $(n=0,60)$; $3,6 \%$ de AAm e $1,0 \%$ de MC $(n=0,78)$; e 7,2 \% de AAm e $1,0 \%$ de MC $(n=0,60)$ apresentaram valores de $D>>D_{\mathrm{H} 2 \mathrm{O}}$. Esse comportamento pode estar relacionado com o tipo de transporte apresentado 
por esses hidrogéis (transporte anômalo, difusão não exclusivamente Fickiana). Assim, o intumescimento nestes hidrogéis depende da relaxação da cadeia polimérica o que aumenta significativamente o valor do coeficiente de difusão.

Se analisarmos os valores de $D$ e de $n$ para um mesmo tipo de hidrogel, porém em diferentes meios de intumescimento nota-se que, de maneira geral, em pH 4,0 e/ou pH 10,0 os valores são menores do que os obtidos em $\mathrm{pH}=7,0$. Isto pode estar relacionado a dois aspectos principais: i) presença de sais no meio de intumescimento; $\mathrm{e}$ ii) ionização dos grupos hidroxilas presentes na MC. Ambos os fatores contribuem significativamente na mudança da pressão osmótica do meio de intumescimento o que, por sua vez, afeta tanto os valores de $D$ quanto os valores de $n$. Foi verificado que este efeito é mais pronunciado em hidrogéis sintetizados a partir de solução com baixa concentração de $\operatorname{AAm}(3,6 \%)$ devido à alta elasticidade das redes tridimensionais dos hidrogéis.

Não foi observada nenhuma tendência pronunciada da constante de difusão $(k)$ com as concentrações de AAm e de MC. De maneira geral, o aumento da concentração de metilcelulose provoca diminuição do valor de $k$ e o aumento de AAm ocasiona incremento na constante cinética. No entanto, os resultados de $D$ mostraram comportamento antagônico. É importante ressaltar que o cálculo de $D$ é realizado com base nos valores de $n$ e $k$ (Equação 3), enquanto que $k$ é obtido diretamente pelo coeficiente linear de $\ln \mathrm{F} v s \ln \mathrm{t}$ (Equação 2), sem levar em consideração o tipo de mecanismo de difusão, representado por $n$, mostrando que o estudo da difusão da água ocorrido através do processo de intumescimento desses hidrogéis é melhor representado matematicamente se for analisada através do parâmetro $D$. Ainda se analisarmos a Equação 3, podemos perceber que $D$ é proporcional ao exponencial do inverso de $n$ e diretamente proporcional a $k$. Assim, os valores de $D$ são muito mais sensíveis à variação de $n$ do que a variação de $k$, o que dificulta a análise dos resultados e a possível correlação entre tais parâmetros cinéticos.

Nas Figuras 3a-c são mostradas as dependências dos valores do expoente difusional $(n)$ obtidos para os três diferentes meios de intumescimento com a concentração de AAm usada para a síntese dos diferentes hidrogéis de PAAm/MC. Naquelas figuras são sinalizadas as regiões onde ocorrem os diferentes mecanismos de difusão (Fickiana e transporte anômalo).

Em todos os meios de intumescimentos investigados foi possível observar que os valores de $n$ diminuem com o aumento da concentração de AAm. Ainda, em ambientes de intumescimentos iônicos e em altas concentrações de AAm (independente do $\mathrm{pH}$ ) a difusão segue o modelo Fickiano. Já em baixas concentrações de AAm, o mecanismo que melhor representa o processo difusional do solvente é o de transporte anômalo.

\section{Correlação das características estruturais $\left(m_{c}, q\right.$ e $\left.v_{e}\right) \operatorname{com} Q_{e q}$}

Os estudos dos parâmetros estruturais de redes dos hidrogéis foram realizados analisando-se três importantes propriedades: massa molar média entre retículos $\left(\mathrm{M}_{\mathrm{C}}\right)$, densidade de reticulação $(\mathrm{q})$, e número de cadeias efetivamente elásticas por unidade de volume $\left(\mathrm{V}_{\mathrm{e}}\right)$. Os valores de cada uma dessas propriedades são mostrados nas Tabelas 2-4.

Pode-se observar na Tabela 2, que para os hidrogéis obtidos em uma dada concentração de AAm e intumescidos em $\mathrm{pH}=7,0$, os respectivos valores de $\mathrm{M}_{\mathrm{C}}$ aumentam bruscamente com o aumento do teor de $\mathrm{MC}$ no hidrogel. Ainda, como esperado, os valores de $\mathrm{M}_{\mathrm{C}}$ estão bem correlacionados com os valores obtidos de $\mathrm{Q}_{\mathrm{eq}}$, ou seja, as redes que apresentam maiores valores de $\mathrm{M}_{\mathrm{C}}$ (mais flexíveis). Por outro lado, para um dado teor de MC, observa-se diminuição significativa nos valores de $\mathrm{M}_{\mathrm{C}}$ quando é aumentada a concentração de AAm na solução formadora do hidrogel. Por exemplo, para $\mathrm{MC}=$
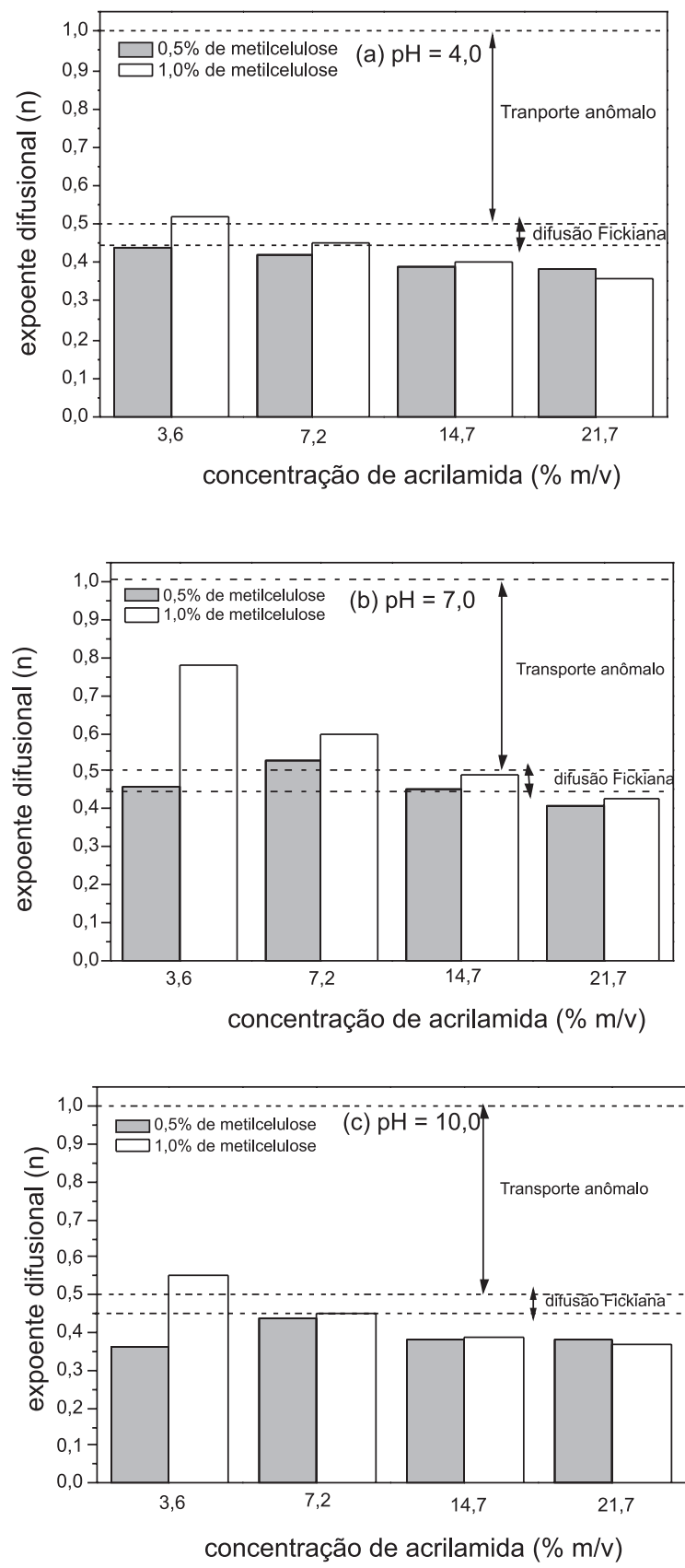

Figura 3. Dependência do expoente difusional (n) com a concentração de AAm usada preparar os hidrogéis intumescido em diferentes condições à 25 ${ }^{\circ} \mathrm{C}$ : a) $\mathrm{pH}=4,0$; b) $\mathrm{pH}=7,0$ e c) $\mathrm{pH}=10,0$

$1,0 \%(\mathrm{~m} / \mathrm{v})$, os valores de $\mathrm{M}_{\mathrm{C}}\left(\mathrm{x} 10^{4} \mathrm{~g} \mathrm{~mol}^{-1}\right)$ são: 43,70; 9,$83 ; 3,07$ e 1,59 quando as concentrações de AAm são 3,6; 7,2; 14,7 e 21,7 \% $(\mathrm{m} / \mathrm{v})$, respectivamente.

A dependência de $\mathrm{M}_{\mathrm{C}}$ com o teor de $\mathrm{MC}$ torna-se menos pronunciada se é utilizada alta concentração de AAm para preparar o hidrogel. Como discutido anteriormente, as redes de hidrogéis formadas a partir da solução com concentração de AAm em 21,7 \% são pouco flexíveis, o que dificulta a expansão e/ou contração dos retículos e, conseqüentemente, a alteração nos valores de $\mathbf{M}_{\mathrm{C}}$.

Quando intumescidos em $\mathrm{pH}=10,0$, os valores de $\mathrm{M}_{\mathrm{C}}$ praticamente seguem a mesma tendência observada que quando intumescidos em $\mathrm{pH}=7,0$, porém com menores valores de $\mathrm{M}_{\mathrm{C}}$. Já em $\mathrm{pH}=4,0$, os valores de $M_{C}$ praticamente não são influenciados pela mudança na concentração de metilcelulose. 
Pode-se observar ainda a existência de uma forte dependência de $\mathrm{M}_{\mathrm{C}}$ com a força iônica do meio de intumescimento para os hidrogéis constituídos de PAAm e MC, desenvolvidos neste trabalho. Por exemplo, os valores de $\mathrm{M}_{\mathrm{C}}\left(\mathrm{x} 10^{4} \mathrm{~g} \mathrm{~mol}^{-1}\right)$ decrescem de 43,70 para 8,58 quando a força iônica é aumentada de 0 para $0,20 \mathrm{~mol}$ $\mathrm{L}^{-1}$. Isto demonstra que a hidrofilicidade desses hidrogéis respondem à mudança da força iônica do meio de intumescimento, i. e., os hidrogéis são sal-responsivos além de serem pH-responsivos, como discutido anteriormente. Essas propriedades, adicionadas à alta velocidade de intumescimento (alta constante de difusão $k$ ), credenciam esses hidrogéis para serem aplicados em diversos campos da agricultura, por exemplo, na liberação controlada de água e insumos agrícolas.

Pelas análises das Tabelas 3 e 4, observa-se que os valores de q e $V_{e}$ seguem perfil antagônico aos valores de $M_{C}$, ou seja, para uma dada concentração de AAm, maiores valores de MC conduzem a menores valores de $\mathrm{q}$ e $\mathrm{V}_{\mathrm{e}}$. Isso já era esperado, pois as equações 4-6 mostram que $M_{C}$ é inversamente proporcional a q e a $V_{\mathrm{e}}$, e que q é diretamente proporcional a $\mathrm{V}_{\mathrm{e}}$ e vice-versa. De maneira geral, hidrogéis de PAAm com maiores teores de $\mathrm{MC}$ apresentam maiores valores de $\mathrm{Q}_{\mathrm{eq}}$ e $\mathrm{M}_{\mathrm{C}}$ e menores valores para os parâmetros q e $\mathrm{V}_{\mathrm{e}}$.

\section{Análise multivariada: planejamento fatorial}

O planejamento fatorial tem sido muito aplicado em pesquisas básicas e tecnológicas e é classificado como um método multivariado, onde pode ser analisado o efeito da variação simultânea de muitas variáveis sobre diferentes propriedades de interesse (respostas). ${ }^{34} \mathrm{O}$ objetivo principal deste trabalho, realizado por meio da construção do planejamento fatorial, foi avaliar a influência desses fatores bem como suas interações sobre as propriedades cinéticas e estruturais (respostas) dos hidrogéis constituídos por PAAm e MC em diferentes formulações.

Tabela 2. Valores de $\mathrm{M}_{\mathrm{C}}\left(10^{4} \mathrm{~g} \mathrm{~mol}^{-1}\right)$ dos hidrogéis obtidos a partir de soluções com diferentes concentrações de AAm e MC intumescidos em meios com diferentes valores de $\mathrm{pH}$ e força iônica

\begin{tabular}{|c|c|c|c|c|c|c|}
\hline Hidrogéis & \multicolumn{3}{|c|}{$\mathrm{pH}$} & \multicolumn{3}{|c|}{ força iônica $\left(\mathrm{mol} \mathrm{L}^{-1}\right)$} \\
\hline $3,6 \%$ AAm & 7,58 & 8,75 & 9,71 & 7,45 & 7,39 & 8,39 \\
\hline $3,6 \%$ AAm e $0,5 \% \mathrm{MC}$ & 10,7 & 24,60 & 18,50 & 7,98 & 9,11 & 11,2 \\
\hline $3,6 \%$ AAm e $1,0 \% \mathrm{MC}$ & 7,36 & 43,70 & 22,30 & 7,23 & 8,02 & 8,58 \\
\hline $7,2 \%$ AAm & 3,06 & 3,43 & 3,30 & 2,97 & 3,23 & 3,20 \\
\hline $7,2 \%$ AAm e $1,0 \% \mathrm{MC}$ & 2,95 & 9,83 & 5,09 & 3,09 & 3,18 & 3,21 \\
\hline $14,7 \%$ AAm & 1,43 & 1,69 & 1,74 & 1,60 & 1,68 & 1,64 \\
\hline $14,7 \%$ AAm e $0,5 \% \mathrm{MC}$ & 1,45 & 2,26 & 2,19 & 1,68 & 1,68 & 1,68 \\
\hline $14,7 \%$ AAm e $1,0 \% \mathrm{MC}$ & 1,56 & 3,07 & 1,86 & 1,59 & 1,63 & 1,68 \\
\hline
\end{tabular}

Tabela 3. Valores de $\mathrm{q}\left(10^{-3} \mathrm{~g} \mathrm{~mol}^{-1}\right)$ dos hidrogéis obtidos a partir de soluções com diferentes concentrações de AAm e MC intumescidos em meios com diferentes valores de $\mathrm{pH}$ e força iônica

\begin{tabular}{|c|c|c|c|c|c|c|}
\hline \multirow[b]{2}{*}{ Hidrogéis } & \multicolumn{3}{|c|}{$\mathrm{pH}$} & \multicolumn{3}{|c|}{ força iônica (mol L-1) } \\
\hline & 4,0 & 7,0 & 10,0 & 0,025 & 0,10 & 0,20 \\
\hline $3,6 \% \mathrm{AAm}$ & 0,94 & 0,81 & 0,73 & 0,96 & 0,96 & 0,85 \\
\hline $3,6 \%$ AAm e $0,5 \%$ MC & 0,67 & 0,29 & 0,39 & 0,89 & 0,78 & 0,63 \\
\hline $3,6 \%$ AAm e $1,0 \% \mathrm{MC}$ & 0,97 & 0,16 & 0,32 & 0,98 & 0,89 & 0,83 \\
\hline $7,2 \%$ AAm & 2,32 & 2,07 & 2,16 & 2,39 & 2,20 & 2,22 \\
\hline $7,2 \%$ AAm e $0,5 \%$ MC & 2,21 & 1,32 & 1,49 & 2,21 & 2,17 & 2,13 \\
\hline $7,2 \%$ AAm e $1,0 \% \mathrm{MC}$ & 2,41 & 0,72 & 1,40 & 2,30 & 2,23 & 2,21 \\
\hline $14,7 \% \mathrm{AAm}$ & 4,97 & 4,22 & 4,08 & 4,45 & 4,24 & 4,32 \\
\hline $14,7 \%$ AAm e $0,5 \% \mathrm{MC}$ & 4,89 & 1,32 & 3,25 & 4,24 & 4,24 & 4,24 \\
\hline $14,7 \%$ AAm e $1,0 \% \mathrm{MC}$ & 4,55 & 2,32 & 3,81 & 4,48 & 4,36 & 4,24 \\
\hline $21,7 \%$ AAm & 6,59 & 6,17 & 5,46 & 6,23 & 5,81 & 5,92 \\
\hline $21,7 \%$ AAm e $0,5 \% \mathrm{MC}$ & 6,21 & 5,36 & 5,12 & 5,81 & 5,81 & 5,62 \\
\hline $21,7 \%$ AAm e $1,0 \% \mathrm{MC}$ & 8,01 & 4,48 & 6,21 & 5,90 & 5,81 & 5,62 \\
\hline
\end{tabular}


Tabela 4. Valores de Ve $\left(10^{19} \mathrm{~mol} \mathrm{~mL}^{-1}\right)$ dos hidrogéis obtidos a partir de soluções com diferentes concentrações de AAm e MC intumescidos em meios com diferentes valores de $\mathrm{pH}$ e força iônica

\begin{tabular}{|c|c|c|c|c|c|c|}
\hline \multirow[b]{2}{*}{ Hidrogéis } & \multicolumn{3}{|c|}{$\mathrm{pH}$} & \multicolumn{3}{|c|}{ força iônica $\left(\mathrm{mol} \mathrm{L}^{-1}\right)$} \\
\hline & 4,0 & 7,0 & 10,0 & 0,025 & 0,10 & 0,20 \\
\hline $3,6 \%$ AAm & 0,87 & 0,76 & 0,68 & 0,89 & 0,90 & 0,79 \\
\hline $3,6 \%$ AAm e $0,5 \%$ MC & 0,62 & 0,27 & 0,36 & 0,83 & 0,73 & 0,59 \\
\hline $3,6 \%$ AAm e $1,0 \% \mathrm{MC}$ & 0,90 & 0,15 & 0,30 & 0,92 & 0,83 & 0,77 \\
\hline $7,2 \%$ AAm & 2,17 & 1,93 & 2,00 & 2,23 & 2,05 & 2,07 \\
\hline $7,2 \%$ AAm e $0,5 \%$ MC & 2,06 & 1,23 & 1,39 & 2,06 & 2,02 & 1,99 \\
\hline $7,2 \%$ AAm e $1,0 \% \mathrm{MC}$ & 2,25 & 0,67 & 1,30 & 2,14 & 2,08 & 2,07 \\
\hline $14,7 \%$ AAm & 4,63 & 3,93 & 3,80 & 4,15 & 3,95 & 4,03 \\
\hline $14,7 \%$ AAm e $0,5 \% \mathrm{MC}$ & 4,56 & 2,93 & 3,03 & 3,95 & 3,95 & 3,95 \\
\hline $14,7 \%$ AAm e $1,0 \% \mathrm{MC}$ & 4,24 & 2,16 & 3,55 & 4,18 & 4,06 & 3,95 \\
\hline $21,7 \%$ AAm & 6,14 & 5,75 & 5,09 & 5,80 & 5,41 & 5,52 \\
\hline $21,7 \%$ AAm e $0,5 \% \mathrm{MC}$ & 5,78 & 4,99 & 4,77 & 5,41 & 5,41 & 5,24 \\
\hline $21,7 \%$ AAm e $1,0 \% \mathrm{MC}$ & 7,47 & 4,18 & 5,78 & 5,50 & 5,41 & 5,24 \\
\hline
\end{tabular}

Nos planejamentos experimentais onde os fatores são analisados em 3 níveis, é comum codificá-los usando (+) para o nível superior, e (-) para o nível inferior. Na Tabela 5 são apresentados os valores reais dos níveis para cada um dos fatores estudados nesse trabalho. Devido aos baixos valores dos coeficientes de regressão linear $\left(\mathrm{R}^{2}\right)$ obtidos para hidrogéis contendo 3,6\% de AAm, tomou-se como nível inferior para essa variável a concentração de 7,2 \%.

Tabela 5. Fatores e níveis utilizados no planejamento fatorial

\begin{tabular}{lcc}
\hline Fatores & nível inferior (-) & nível superior $(+)$ \\
\hline A & 7,2 & 21,7 \\
B & 0,5 & 1,0 \\
C & 4,0 & 10,0 \\
\hline
\end{tabular}

$\mathrm{A}=\operatorname{AAm}(\% \mathrm{~m} / \mathrm{v}), \mathrm{B}=\mathrm{MC}(\% \mathrm{~m} / \mathrm{v})$ e $\mathrm{C}=\mathrm{pH}$.

Na Tabela 6 são apresentados os valores obtidos nos diferentes experimentos para as propriedades estruturais e cinéticas com as respectivas significâncias obtidas fixando-se o limite de confiança em $95 \%$. A determinação da significância de cada efeito foi obtida por análises de tabelas de análise de variância (ANOVA). Para interpretar os dados experimentais utilizou-se o programa computacional Design Expert ${ }^{\circledR}$. Os resultados do planejamento fatorial $2^{3}$ foram expressos por meio do modelo matemático dado pela Equação 7:

$\mathrm{y}=\mathrm{b}_{0}+\mathrm{b}_{\mathrm{A}} \mathrm{A}+\mathrm{b}_{\mathrm{B}} \mathrm{B}+\mathrm{b}_{\mathrm{C}} \mathrm{C}+\mathrm{b}_{\mathrm{AB}} \mathrm{A}^{* \mathrm{~B}}+\mathrm{b}_{\mathrm{AC}} \mathrm{A}^{* \mathrm{C}}+\mathrm{b}_{\mathrm{BC}} \mathrm{B}^{*} \mathrm{C}+\mathrm{b}_{\mathrm{ABC}} \mathrm{A}^{*} \mathrm{~B} * \mathrm{C}$

onde y é a resposta, $\mathrm{b}_{0}$ é a média dos fatores na resposta y, $\mathrm{b}_{\mathrm{i}}$ são os fatores principais, $b_{i j}$ e $b_{i j k}$ são os produtos de interações secundárias e terciárias dos fatores A, B e C. ${ }^{35}$ No presente trabalho, o fator A corresponde à concentração de acrilamida $(\% \mathrm{~m} / \mathrm{v})$, o fator $\mathrm{B}$ à concentração de metilcelulose $(\% \mathrm{~m} / \mathrm{v})$, e o fator $\mathrm{C}$ ao $\mathrm{pH}$.

A significância estatística obtida para os efeitos principais e suas interações relativas às propriedades cinéticas e estruturais dos diferentes hidrogéis estudados também pode ser visualizada através de análises de gráficos normais representados na Figura 4 e 1S (Material Suplementar).

Foi possível observar por planejamento fatorial que o fator AAm contribui negativamente para os valores de $\mathrm{Q}_{\mathrm{eq}}$ e o fator $\mathrm{pH}$ contribui positivamente. Assim, mantendo a variável AAm em seu nível inferior e pH em seu nível superior, obtem-se hidrogéis com alta absorção de água, alto valor de Q. Do mesmo modo, ao passar do nível inferior para o nível superior dos fatores AAm e pH, observa-se diminuição dos valores das propriedades cinéticas $n$ e $D$. Sendo que os valores apresentados para a variável $\mathrm{pH}$ encontra-se no limite da significância. Já à alteração no nível do fator MC (do nível inferior para o nível supe-

Tabela 6. Valores dos fatores, modelo matemático e significância para os diferentes níveis obtidos para as propriedades cinéticas e estruturais

\begin{tabular}{|c|c|c|c|c|c|}
\hline Propriedades & Média & A & B & $\mathrm{C}$ & Modelo matemático * \\
\hline $\mathrm{Q}_{\mathrm{eq}}(\mathrm{g} / \mathrm{g})$ & 1751,25 & $-536,25$ & $-16,25 * *$ & 176,25 & $\mathrm{Q}_{\mathrm{eq}}=1751,25-536,25 \mathrm{~A}+176,25 \mathrm{C}$ \\
\hline $\mathbf{n}$ & 0,41 & $-0,034$ & $0,0013 * *$ & $-0,0038$ & $\mathrm{n}=0,41-0,034 \mathrm{~A}+0,0038 \mathrm{C}-0,0088 \mathrm{AB}$ \\
\hline$D\left(10^{-5} \mathrm{~cm}^{2} \mathrm{~s}^{-1}\right)$ & 1,78 & $-0,73$ & $-0,01 * *$ & $-0,045$ & $\mathrm{D}=1,78-0,73 \mathrm{~A}-0,045 \mathrm{C}+0,33 \mathrm{~A} \mathrm{~B} C$ \\
\hline $\mathbf{k}\left(\mathbf{s}^{-1}\right)$ & 0,15 & 0,014 & $-0,027$ & $-0,0099$ & $\mathrm{k}=0,15+0,014 \mathrm{~A}-0,027 \mathrm{~B}-0,0099 \mathrm{C}$ \\
\hline $\operatorname{Mc}\left(10^{4} \mathrm{~g} \mathrm{~mol}^{-1}\right)$ & 2,57 & $-1,43$ & $-0,056 * *$ & 0,53 & $\mathrm{Mc}=2,57-1,43 \mathrm{~A}+0,53 \mathrm{C}-0,40 \mathrm{~A} \mathrm{C}$ \\
\hline $\mathrm{q}\left(10^{-3} \mathrm{~g} \mathrm{~mol}^{-1}\right)$ & 4,13 & 2,26 & 0,37 & $-0,58$ & $q=4,13+2,26 A+0,37 B-0,58 C+0,35 A B$ \\
\hline Ve $\left(10^{19} \mathrm{molmL}^{-1}\right)$ & 3,85 & 2,10 & 0,35 & $-0,54$ & $\mathrm{~V}_{\mathrm{e}}=3,85+2,10 \mathrm{~A}+0,35 \mathrm{~B}-0,54 \mathrm{C}+0,33 \mathrm{AB}$ \\
\hline
\end{tabular}

* Modelos obtidos através do planejamento fatorial considerando apenas os valores estatisticamente significativos.

** Valores em vermelho são estatisticamente não significativos. 

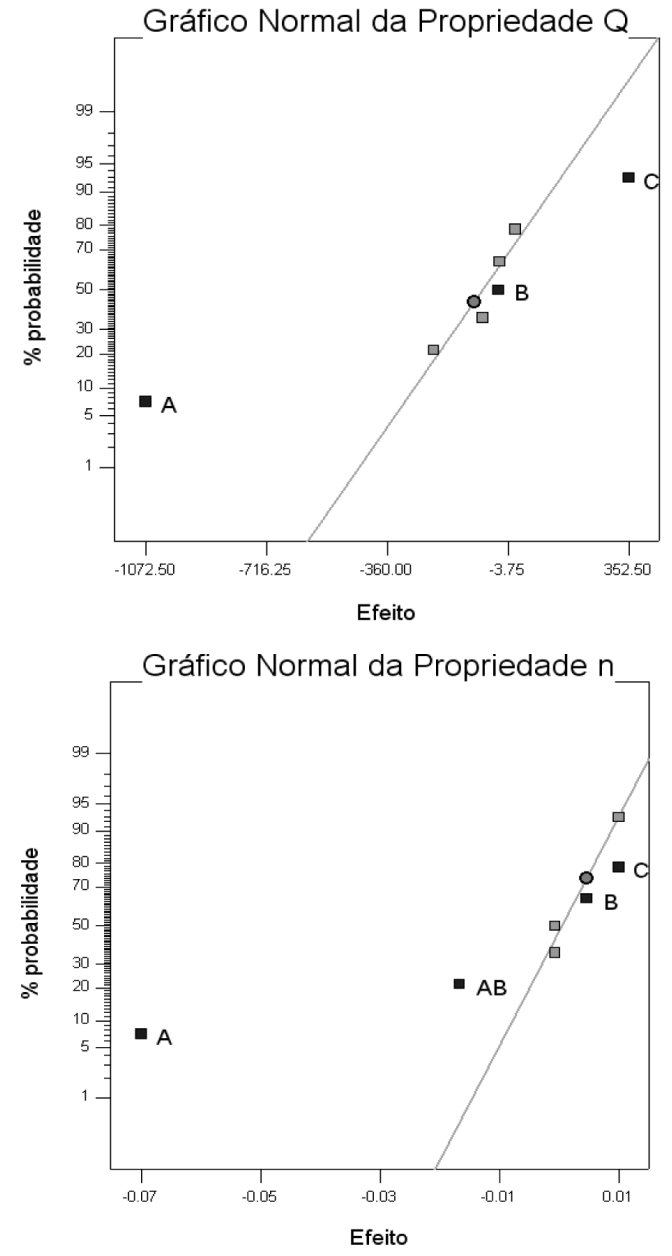

Figura 4. Gráficos normais dos efeitos mostrados na Tabela 6 correspondentes as propriedades hidrofilica - $Q$ e cinética - $n$ indicando a significância estatística de cada efeito principal e suas interações.

rior, ou vice-versa) não proporciona mudança significativa nos valores das propriedades mencionadas. Indicando assim que a concentração de metilcelulose a $0,5 \%(\mathrm{~m} / \mathrm{v})$ é suficiente para causar o mesmo efeito em tais propriedades para os respectivos hidrogéis contendo $1,0 \%$ $(\mathrm{m} / \mathrm{v})$ desse mesmo polímero. As análises efetuadas para a constante cinética $k$ mostra a mesma tendência discutida e mostrada anteriormente, ou seja, aumento de $k$ com o aumento de AAm, diminuição de $k$ com o incremento de $\mathrm{MC}$ e uma pequena diminuição ao variar o pH da solução de intumescimento de 4,0 para 10,0.

Pelas análises dos efeitos mostrados na Tabela 6 observou-se que a variável acrilamida foi mais significativa que as variáveis metilcelulose e $\mathrm{pH}$. Sendo que a mesma contribui positivamente na resposta $q$ e $V_{e}$ e negativamente na propriedade estrutural $M c$. Como esperado, o efeito das variáveis apresentam comportamentos antagônicos para as propriedades estruturais: $M_{C}$ inversamente a $q$ e $V_{e}$, e a propriedade $q$ diretamente a $V_{e}$ ou vice-versa.

\section{CONCLUSÕES}

Foi possível obter, com sucesso, hidrogéis a partir da polimerização do monômero acrilamida (AAm), sendo a reticulação feita pela concomitante copolimerização do $\mathrm{N}, \mathrm{N}^{\prime}$-metilenobisacrilamida (MBAAm). Como o processo de gelificação ocorreu na presença do polissacarídeo biodegradável metilcelulose (MC), houve efetiva incorporação de MC na rede dos diferentes hidrogéis. Com a incorporação de MC no hidrogel houve variação principalmente na hidrofilicidade dos géis. Foram feitas diferentes formulações variando-se a quantidade de AAm e de MC na soluções formadoras dos hidrogéis.

Os valores de grau de intumescimento, $\mathrm{Q}_{\mathrm{eq}}$, (que está diretamente relacionado com a hidrofilicidade) variaram entre 1100-9200\%. Este parâmetro é fortemente dependente dos fatores: concentrações de AAm e de MC da solução onde o hidrogel foi preparado e do $\mathrm{pH}$ do meio de intumescimento. Ainda, o fato da incorporação de MC deixar a matriz polimérica mais hidrofílica amplia o campo de aplicação dos hidrogéis de PAAm/MC.

A partir de curvas de cinéticas de intumescimento (feitas a $25^{\circ} \mathrm{C}$ ) dos diferentes hidrogéis estudados neste trabalho foram determinados os valores do expoente difusional $(n)$, constante de difusão $(k)$ e do coeficiente de difusão $(D)$. O transporte do solvente para o interior do hidrogel é fortemente influenciado pelas concentrações de AAm e MC usadas na síntese do hidrogel. Tanto $n$, quanto $D$ são diretamente proporcionais ao grau de intumescimento $\left(\mathrm{Q}_{\mathrm{eq}}\right)$ dos hidrogéis.

As propriedades estruturais [massa molar média entre retículos $\left(\mathrm{M}_{\mathrm{C}}\right)$, densidade de reticulação (q), e número de cadeias efetivamente elásticas por unidade de volume $\left(\mathrm{V}_{\mathrm{e}}\right)$ ] podem ser controladas pelo ajuste da concentração de AAm, MC e pH da solução externa de intumescimento.

Devido à alta hidrofilicidade dos hidrogéis sintetizados e caracterizados nesse trabalho, o que permite rápida absorção de água, estes materiais podem ser considerados como extremamente promissores para serem aplicados em diferentes campos na agricultura, destacando a liberação controlada de água e/ou pesticidas de âmbito geral. O uso destes materiais possibilita controlar a liberação de pesticidas por meio do controle da hidrofilicidade dos mesmos através da variação entre a relação acrilamida / metilcelulose na solução formadora dos hidrogéis.

\section{MATERIAL SUPLEMENTAR}

No material suplementar, disponível gratuitamente em http:// quimicanova.sbq.org.br na forma de arquivo PDF, estão apresentados na Figura $1 \mathrm{~S}$ os gráficos normais dos efeitos mostrados na Tabela 6 correspondentes as propriedades cinéticas (D e k) e estruturais (Mc, q e Ve) indicando a significância estatística de cada efeito principal e suas interações.

\section{AGRADECIMENTOS}

Os autores agradecem ao Conselho Nacional de Desenvolvimento Científico e Tecnológico (CNPq) pelas bolsas de doutorado e produtividade em pesquisa, Embrapa (Programa Labex e Projeto MP1), FINEP/LNNA pelo suporte financeiro.

\section{REFERÊNCIAS}

1. Onoda-Yamamuro, N.; Yamamuro, O.; Inamura, Y.; Nomura, H.; Phys. B 2007, 393, 158.

2. Ott, E. High polymers - cellulose and cellulose derivatives, Interscience Publishers Inc: New York, 1943.

3. Hermans, P. H.; Weidinger, A.; J. Appl. Phys. 1948, 19, 491.

4. Benamer, S.; Mahlous, M.; Boukrif, A.; Mansouri, B.; Larbi Youcef, S.; Nucl. Instrum. Methods Phys. Res., Sect. B 2006, 248, 284.

5. Rosiak, J. M.; Ulanski, P.; Radiat. Phys. Chem. 1999, 55, 139.

6. Krušić, M. K.; Filipović, J.; Polymer 2006, 47, 148.

7. Kiritoshi, Y.; Ishihara, K.; Polymer 2004, 45, 7499.

8. Guo, B. -L.; Gao, Q. -Y.; Carbohydr. Res. 2007, 342, 2416.

9. Feng, X.; Pelton, R.; Macromolecules 2007, 40, 1624.

10. Yi, J. -Z.; Zhang, L. M.; Bioresour. Technol. 2008, 99, 2182.

11. Şolpan, D.; Kölge, Z.; Radiat. Phys. Chem. 2006, 75, 120.

12. Kioussis, D. R.; Kofinas, P. P.; Polymer 2005, 46, 10167. 
13. Willems, H. P. L.; Berry, D. F.; Environ. Sci. Technol. 1996, 30, 2148.

14. Jeon, O.; Song, S. J.; Lee, K. -J.; Park, M. H.; Lee, S. H.; Hahn, S. K.; Kim, S. K.; Kim, B. -S; Carbohydr. Polym. 2007, 70, 251.

15. Emileh, A.; Vasheghani-Farahani, E.; Imani, M.; Eur. Polym. J. 2007, 43, 1986.

16. Abd Alla, S. G.; El-Din, H. M. N.; El-Naggar, A. W. M.; Eur. Polym. J. 2007, 43, 2987.

17. Unal, B.; Hedden, R. C.; Polymer 2006, 47, 8173.

18. Guilherme, M. R.; da Silva, R.; Rubira, A. F.; Geuskens, G.; Muniz, E. C.; React. Funct. Polym. 2004, 61, 233.

19. Morita, T., Assumpção, R.M.V.; Manual de Soluções, Reagentes e Solventes. Editora Edgard Blucher: São Paulo, 2001.

20. Teijón, C.; Olmo, R.; Dolores Blanco, M.; Teijón, J. M.; Romero, A.; J. Colloid Interface Sci. 2006, 295, 393.

21. Spinks, G. M.; Lee, C. K.; Wallace, G. G.; Kim, S. I.; Kim, S. J.; Langmuir 2006, 22, 9375.

22. Reddy, T. T.; Lavenant, L.; Lefebvre, J.; Renard, D.; Biomacromolecules 2006, 7, 323.

23. Sriamornsak, P.; Thirawong, N.; Weerapol, Y.; Nunthanid, J.; Sungthongjeen, S.; Eur. J. Pharm. Biopharm. 2007, 67, 211.
24. Ritger, P. L.; Peppas, N. A.; J. Controlled Release 1987, 5, 37.

25. Kundakci, S.; Uzum, O. B.; Karadag, E.; React. Funct. Polym. 2008, 68 , 458.

26. Caykara, T.; Kiper, S.; Demirel, G.; Eur. Polym. J. 2006, $42,348$.

27. Lin, Z.; Wu, W.; Wang, J.; Jin, X.; React. Funct. Polym. 2007, 67, 789.

28. Bajpai, A. K.; Giri, A.; Carbohydr. Polym. 2003, 53, 271.

29. Amsden, B.; Macromolecules 1999, 32, 874.

30. Flory, P. J.; Rehner, J. J.; J. Chem. Phys. 1943, 11, 521.

31. Aouada, F. A.; de Moura, M. R.; Fernandes, P. R. G.; Mukai, H.; da Silveira, A. C. F.; Itri, R.; Rubira, A. F.; Muniz, E. C.; Eur. Polym. J. 2006, 42, 2781.

32. Lynch, I.; Dawson, K. A.; J. Phys. Chem. B 2004, 108, 10893.

33. Serra, L.; Doménech, J.; Peppas, N. A.; Biomaterials 2006, 27, 5440.

34. Bruns, R. E.; Barros Neto, B.; Scarmínio, I. S.; Como fazer experimentos. Pesquisa e desenvolvimento na ciência e na indústria, Editora da Unicamp: Campinas, 2001.

35. Aouada, F. A.; de Moura, M. R.; Campese, G. M.; Girotto, E. M.; Rubira, A. F.; Muniz, E. C.; Polímeros 2008, 18, 126. 


\section{CORRELAÇÃO ENTRE PARÂMETROS DA CINÉTICA DE INTUMESCIMENTO COM CARACTERÍSTICAS ESTRUTURAIS E HIDROFÍLICAS DE HIDROGÉIS DE POLIACRILAMIDA E METILCELULOSE}

Fauze A. Aouada

Departamento de Química, Universidade Federal de São Carlos, 13560-905 São Carlos - SP, Brasil

Edvani C. Muniz

Departamento de Química, Universidade Estadual de Maringá, 87020-900 Maringá - PR, Brasil

Carlos M. P. Vaz e Luiz H. C. Mattoso*

Laboratório Nacional de Nanotecnologia para o Agronegócio, Embrapa Instrumentação Agropecuária,

13560-970 São Carlos - SP, Brasil
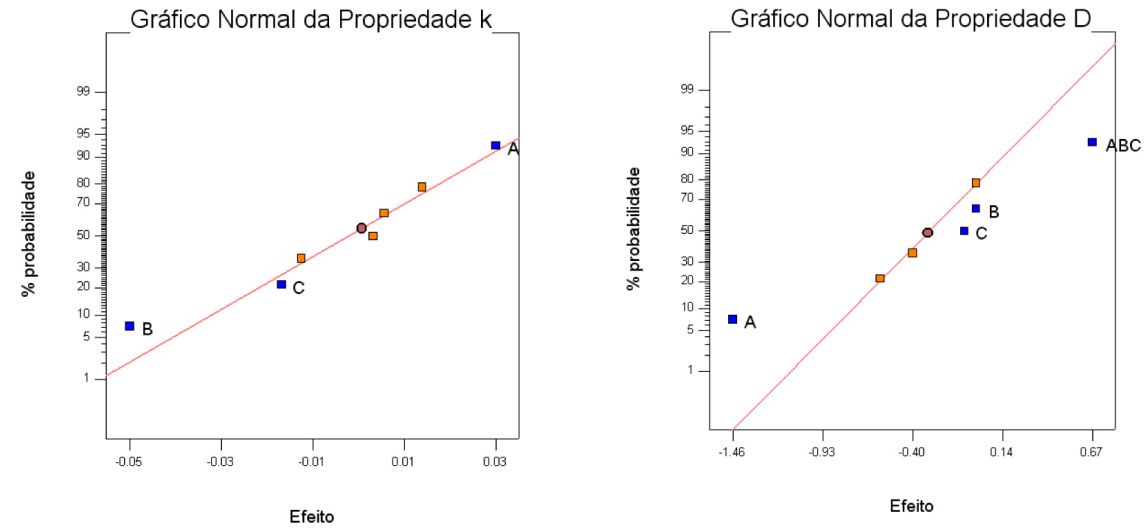

Efeito

Efeito
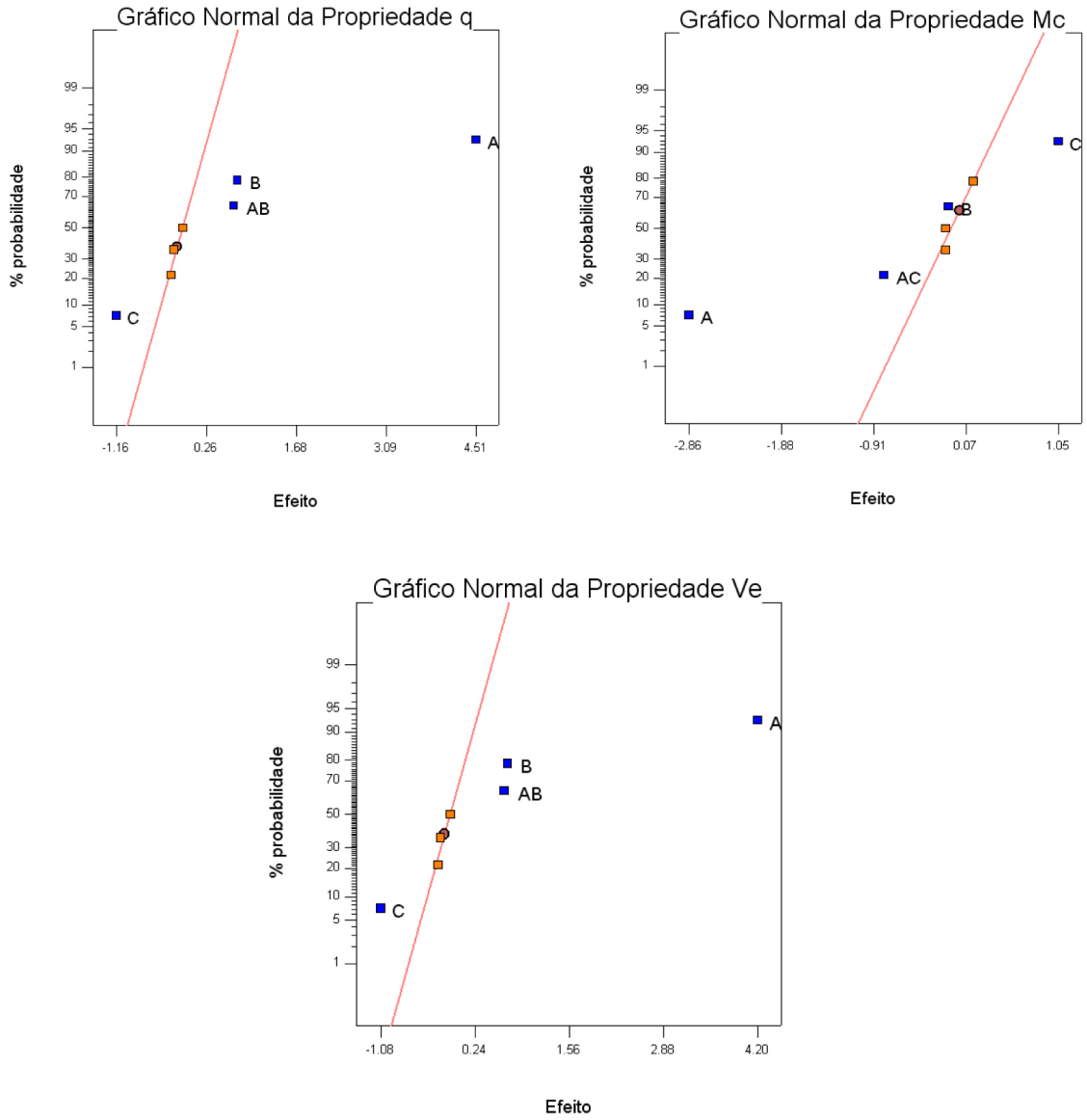

Figura 1S. Gráficos normais dos efeitos mostrados na Tabela 6 correspondentes as propriedades cinéticas (D e k) e estruturais (Mc, q e Ve) indicando a significância estatística de cada efeito principal e suas interações

*e-mail: mattoso@cnpdia.embrapa.br 